\title{
On the Nature of Mind.*
}

\author{
By Dr. C. S. Myers, C.B.E., F.R.S.
}

$\mathrm{P}^{\mathrm{s}}$ SYCHOLOGY was given the status of an independent Section, with the approval of the Sections of Physiology and Education, at the Cardiff meeting of the British Association in 1920. The new Section met for the first time in 1921 under the presidency of Prof. Lloyd Morgan. Thus the present centenary meeting marks also the completion of ten years' existence of our Section. I can vividly recall the doubts which were expressed, not so much in words, as in general attitude, by the Committee of Recommendations of this Association when in 1920 it was asked to consider the formation of a separate Section of Psychology. Such hesitation was probably based on several grounds, not wholly on any one of them. But some of these grounds have lost much of their force to-day, because of the pronounced change that has since taken place in the attitudes and beliefs which were in vogue among physicists of that time. No physicists would then have dared, as now, to cast doubt on the sole sway of determinism in the physical world. None of them would then have suggested, as now, the impossibility of predicting what any individual atom (or still smaller individual entity) will do next. None would have questioned, as now, the universal truth of the second law of thermodynamics or of the principle of conservation of energy. None would have ventured, as now, to suppose that electrons change in the very act of becoming known to us, and that therefore the mental factor is ultimately inseparable from physical investigations. None would then have dared, as now, to conjecture that particles of matter correspond in their properties to certain group waves of the ether, the constituent waves of which, travelling at an enormous speed, 'guide' and 'direct' the group waves without any energy of their own.

Psychology has been similarly bereft of reality by the operations of mathematicians, present and past. The earliest example of this was the derivation by Fechner of the 'law' which now bears his name, that the intensity of a sensation is proportional to the logarithm of the magnitude of its stimulus. This statement was deduced by purely mathematical procedure from Weber's law that just appreciable differences between sensations depend on a constant ratio between the magnitudes of their respective stimuli. Weber's law, however, was based on direct observation and experiment; whereas Fechner's ' law' was the outcome of purely mathematical calculations which not only neglected a constant appeal to the 'reality' of experience, but also ran actually counter to it-neglectful, for example, of the 'facts' (i) that Weber's law holds for only moderate magnitudes of stimuli; (ii) that from the point of view of conscious experience it implies a single experience of difference, not a difference between two separate experiences; and

* From the presidential address to Section $\mathbf{J}$ (Psychology) of the British Association, delivered in London on Sept. 28. that (iii) from the same point of view we are quite unwarranted in adding together or subtracting from one another two intensities of sensation or two sensation differences as such.

Elsewhere in his treatment of psychological data, as in his treatment of physical data, the mathematician has arrived at results that can directly be neither verified nor rejected by conscious experience. The establishment of 'general mental factors', involved in and influencing the performance of various mental tests and other processes, affords us another example. By mathematical operations on experimental data we can, it is claimed, deduce the existence of such general factors. But from the strictly psychological point of view the nature of these factors cannot be interpreted; for we are unable to appeal to direct experience to ascertain what these factors are. At best their significance in terms of actual experience can only be conjectured by abstraction and imagination ; or it is expressible only in terms of general behaviour. At worst, as in the case of $g$ (the socalled 'general intelligence'), we are quite ignorant of their psychical nature. We cannot hope for direct psychological evidence as to the precise mental nature of such mathematically deduced 'factors'.

\section{Psychology aNd Behaviour.}

The escape from such difficulty on the physical side-so as to avoid dethronement of the literally divine claims which some mathematicians have made for the fundamental truth of their own science - is to regard the universe as constituting a vast nexus of ultra-physical and mathematical necessities and probabilities, only some of which can become physical through the further operation of the human mind. We might, perhaps, adopt a corresponding attitude towards some of the subjectively unverifiable conclusions arising from the applications of mathematics to psychological data.

In psychology an additional difficulty confronts us. We have to recognise that the data with which the 'mathematical psychologist' operates are not measurements of the fundamental subject matter of psychology-conscious mental processes. (Nor can they probably be measurements of unconscious processes, so long as the latter are regarded as mental in character.) For mental processes are not directly measurable: we can grade a series of conscious experiences according to their degree or amount, say of hue, brightness, loudness, pitch, temperature, extent, duration, clearness, pleasantness, etc. : we can say that one member of such a series has more or less of any one such character or quality than another member, or that the difference between two members of a series in respect to any one of these characters or qualities is greater or less than, or equal to, the difference between two other members. But the psychologist can only measure the amount of any conscious experience

No. 3235, Vol. 128] 
indirectly-either by reference to behaviour, that is, to the organism's physical response or expression, or by reference to the physical character of the relevant stimuli, in terms of objective standards of number, space, and time which are immediately independent of actual subjective experience.

Let us remember, then, that when we are attempting to measure any mental ability or character or quality by means of a test, or series of tests, we are not directly measuring that mental ability or character or quality, but only the corresponding stimulus or the outward response or expression by which that mental ability or character or quality is manifested. We are, no doubt, justified in assuming a broad correlation between the speed, accuracy, amount, etc., of the response or expression of a mental ability or character or quality and the degree in which that mental ability or character or quality is present. Even this broad assumption, however, is sometimes unjustifiable, as in the case where too much of a given mental ability or character or quality may lead to a deterioration, and no longer to an improvement, in the corresponding performance. But we are certainly never justified in assuming that we can measure accurately any mental process by measuring its objective response -that, for example, twice the amount of the response necessarily means twice the quantity of the mental process of which the response is the expression. All that we are measuring is behaviour - that is to say, something largely on the efferent side, something largely physical and indescribable in terms of pure immediate experience, involving a complex of factors many of which, indeed, may be remote from those which we commonly believe we are measuring; whereas what we ultimately aim at dealing with in psychology is experience - the meeting point of the afferent and efferent sides.

In fact, then, the ' behaviourists' are quite right when they insist that scientific measurement is applicable only to the behaviour of the organism. Where they are quite wrong is in their assumption that conscious processes must necessarily be ousted from scientific psychology, because measurement is excluded; the truth being that, even where measurement is excluded, the possibilities of systematic observation and experiment still remain. Natural science has surely a function wider than that of merely reducing its subject matter to units of space and time. Highly valuable and deserving of the utmost encouragement as is the measurement of behaviouristic data, however helpful be the light they may ultimately throw on mental processes and their general characters, however wider be mental processes than the range of mere conscious experience, the scientific study of the mind by direct observation and experiment is never to be discountenanced or discarded.

\section{SELF-ACTIVITY.}

Just as experimental physics patiently pursues its researches into Nature, heedless of such mathematical conclusions as are not amenable to verification by experiment, so experimental psychology must realise that its progress is not primarily dependent on, however much assistance it may receive from, the work of those who fail to recognise that the fundamental subject matter of psychology is conscious experience, not conduct. Now conscious experience can only be enjoyed by the active self, namely, the 'individual' (that is, undivided) mental activity of the entire living organism. It is the fundamental function of such self-activity-by recourse to past experiences, by receiving present experiences, by foreseeing future experiences and by creating new experiences - to select from alternative responses and from alternative environments those which are most advantageous to the ever-evolving and developing organism ; to secure the most suitable movements and environments and thus to help in the evolution of the organism are the prime objects of consciousness. Where, as in plants, mobility and plasticity are at a minimum, self-activity and consciousness are inappreciable. Self-activity is to be regarded as the highest, unitary integration of the directive mental (conscious and unconscious) activity of the organism.

\section{Derivation of Presentations (or Contents) From FenLings.}

Self-activity and its inherent consciousness may presumably be traced back to a stage where self and not-self are but just distinguishable. At this remote stage in animal evolution there can scarcely have been more than a differentiation of selfactivity into 'acts' of the self and 'modifications' of the self. These modifications of the self became early differentiated into $(a)$ those which are due to internal happenings within the organism, and $(b)$ those which are due to more variable external happenings in its environment, and later into $(a)$ those which we come to recognise as 'affects' of the self and 'feeling tones', and $(b)$ those which come to be regarded as 'presentations' to the self or as 'contents' of the self's consciousness. Sensations, perceptions, memories, and thoughtsall that we finally come to recognise as conscious presentations to the self-have been differentiated (onto- and phylo-genetically) from modifications of the self : instead of being feelings of the self, they have become contents of consciousness.

We end by 'projecting ' certain of these original feelings. The external 'objects' of our perception have been separated from or carved out of originally vague external 'situations', of which we or our remote ancestors were first conscious merely as diffuse modifications or feelings of the self. So, too, any colour or sound comes to be regarded no longer as a self-feeling but as a something projected and existing outside us. The degree to which such projicience and presentation is carried out varies with different sensations: colours clearly have a projected, apparently independent, existence ; sounds, smells, tastes, hardness, and temperature are only imperfectly projected; the painful prick of a pin and our sensations of movement, though not projected, are nevertheless regarded as ' presentations 'to the self; whereas our experiences of visceral sensations are scarcely even presented:

No. 3235, VoL. 128] 
they seem almost as clearly modifications of the self as are our emotions and other affects.

\section{Origin of Acts aNd Contents.}

This difference between the acts and the contents of consciousness - between the conscious acts of apprehending, recalling, deciding, inferring and what is consciously apprehended, recalled, decided, inferred-is a most important one. It is exemplified in the two kinds of memory which are distinguishable. On one hand, we may recall the separate acts of the self, say, in the course of solving a problem or of acquiring some specific skill; these are individually unique and only individually revocable. On the other hand, we may recall the generalised contents of our consciousness, that is, of presentations we have received by a repetition of such acts, for example, in learning a prose passage or series of skilled movements.

I would suggest that the distinction between conscious acts and contents has come about with the gradual differentiation of higher and lower levels of mental activity-and in the following manner. There is no awareness of self-activity when we sense a colour or a temperature, or when we perceive a familiar object, or when an idea 'occurs' to us. Our sensations, our perceptions, and many of our thoughts and ideas are, I suggest, the unconscious 'acts' of relatively lower mental levels. But when these lower-level 'acts' are accompanied and received by the self-activity of the highest levels, they become ipso facto 'presentations' to the self. A loud noise to which we are impelled to attend or an idea which 'occurs to the mind ' is not a conscious presentation (or content of consciousness) until the self receives it.

I suggest that such differentiation of higher and lower levels has never occurred to the same extent in the case of our kinæsthetic and, especially, conæsthetic sensations and in the case of our feelings (which depend on a more primitive, thalamic, activity) : we fail, therefore, to objectify them immediately as presentations, and they continue to be received in their primordial undifferentiated state.

\section{Unconscious Direction and Purpose.}

The self is the highest controlling and directing power. The orders which it consciously gives and the efforts which it consciously makes may, once started, continue to be carried on unconsciously, that is, without the conscious participation of the self. Thus we may consciously but vainly try to recall some past experience or to solve some difficult problem; and after giving up the effort, this directive activity may still persist unconsciously until suddenly the forgotten object, or the abandoned solution, suddenly flashes full-born and unbidden into the self's consciousness. So, too, we may go to sleep determined to wake up at a given hour, or we may accept, in the hypnotic state, a decision to carry out some prescribed act on the lapse of a prescribed period of time after emerging from that state; and at the ordained moment the sleeper wakes, or an uncontrollable impulse is felt to perform the suggested act.
Not only is purposive activity not limited to the duration of conscious activity but also it need not originate there. The inspirations of genius and the intuitive judgments and decisions which, crude though they may be before submission to the self's judgment, arise apparently from the 'depths' of the mind with impulsive force and compelling conviction afford striking examples of this fact. The well-known improvements in learning which continue after we have ceased to practise, so that it has been said of us that we learn to skate in summer and to swim in winter, are further examples of such activity - whether or not we choose to ascribe such improvement to the gradual disappearance of adverse initial inhibitions or to the direct strengthening ('consolidation') of acquired integrations (or associations).

Further, the self is continually being played upon both by the impulsive and by the perseverating forces of lower mental systems. They struggle, not less than the self, for their own existence and for their own lower 'self-ish' ends. Where they are modified by inhibition (or repression), it is only to ensure general harmony and general compatibility. Inhibition is not to be viewed as a mere act of passive drainage of energy from one mental constellation to another, but as an active repressive force against which the inhibited constellation ever tends to rebel in its endeavour to gain somehow or another liberty of action, in some lower degree purposeful and directive.

\section{Cerebral Localisation of Consciousness.}

While consciousness always implies self-activity, and while the self is to be regarded as the expression of the highest level of mental activity, we must guard against the notion that such high-level activity implies a narrowly limited zone of mental processes. On the contrary, it implies a wide sphere of activity rather than a punctate, pineal, gland-like soul. It follows, therefore, that we cannot hope to localise any act or any content of consciousness in one small region of nervous substance. Afferent-efferent localisations of function undoubtedly occur-regions where the incoming impulses become deflected to outgoing processes : our knowledge of the physiology and structure of the spinal cord clearly points to this. Sensorimotor localisations may, in a sense, be said to exist similarly in the brain. The occipital region of the cerebral cortex, for example, is concerned with vision. But because vision ceases when the area striata in this region of the cortex is injured, we are not justified in saying that this area is the seat or centre of our visual consciousness. All that we are warranted in concluding is that it is essential for our visual consciousness, that without it vision is impossible-a very different statement.

Once again, let me repeat, consciousness implies self-activity. There are no separate loci for different kinds or modes or qualities of consciousness. The nervous system and the system of self-activity work as a widespread unity. Different regions of the brain are more particularly concerned in giving rise to certain kinds of consciousness. The thala- 
mus, for example, is especially concerned with the emotional consciousness; but we are not justified in calling it the seat or centre of such consciousness.

We are, in fact, neither warranted in supposing that there are definite seats or centres of sensation or emotion, nor justified in supposing that our manifold percepts, images, or ideas each have their seat in different narrowly localised centres of the brain. A similar truth holds for the association (or integration) of such experiences. We can mentally picture an integration of two "patterns' of conscious activity occurring when two experiences $a$ and $b$ follow one another repeatedly, so that when $a$ is later given, $b$ (or rather the whole $a-b$ ) recurs; but neurologically we can form no simple corresponding picture of two collections of nerve cells being associated together. We have no evidence to support such cerebral localisation of association areas; indeed such experimental evidence as we have is against it.

Even if there were no evidence pointing in one direction or the other, how could such localisation of memories and habits possibly occur? Consider the babe that is learning to associate its mother with the satisfaction of its hourly wants. Its mother is never twice the same-now in one dress or facial expression, now in another ; and the visual image of the mother received by the retina is never twice the same-for example, sometimes the mother is very near, sometimes farther off; sometimes the image falls on one part of the retina, sometimes on another. How can we imagine, then, any definite collection of retinal or cortical nerve cells responsible for developing the image of 'mother'? What develops is surely rather a 'meaning '-a generalisation of images, 'standing for' something, that is, for the assuaging of certain needs, for the execution of a wide range of adjustments of the infant.

Relationships and meanings are therefore the all-important mental acquirements. The acquisition of such relationships is shown, for example, in the many experiments conducted on a large variety of species, high and low in the animal scale, where by long practice the organism is trained to enter $B$, the brighter of two alternative compartments, $A$ and $B$, in order to reach its food. When later, in place of $A$ and $B, B$ and $C$ are substituted, $C$ being now brighter than $B$, does the animal go to $B$ to which it had previously been trained to go ? Generally, no. It enters the $C$ compartment. That is to say, it has learnt to enter not a particular compartment, but the brighter of any two compartments. It has not learnt to select a 'particular' object. It has learnt a 'relation'. Surely evidence of this kind is contrary to any atomistic localisation of individual mental functions in separate cerebral areas.

\section{Relation between Directive and Mechanical Activity.}

The fundamental purpose of consciousness is to enable the self to preserve the organism by guidance and direction-by the formation and satisfaction of ends and values. As in the evolution of living species something far more is involved than the mere blind running down - hill of a wound - up mechanism, so in the mental and bodily life of each organism the physical conceptions of 'entropy' and of mechanical energy are inadequate. On the physical side we can form no conception of the mode of working, throughout life and mind, of anything resembling Clerk Maxwell's directive 'demon'. Physiologically, that is to say, physically, the brain-worker should need food with a far lower caloric value than he actually takes and requires for the successful maintenance of his purely mental activities. But, in fact, mental work appears to make far greater demands on metabolism than it should according to purely physical considerations of the expenditure of mechanical energy.

At present we can form no conception of the nature of the undoubted connexion between chemical metabolism and direction in the living organism -between senility of body and senility of mind, between the rise and decay of procreativeness and the rise and decay of the creativeness of genius. At present we can form no bridge between mechanical and creative, directive activity. We can only say that both activities are essential to a conception of the evolution and working of life and mind. Mental activity involves a quintessence of the non-mechanical, directive activity of life ; and consciousness is but that activity raised to its highest power. Even lower-level mental and neural systems, even the activities of the lowest living organisms, are characterised by unconscious creation, direction, guidance, and purpose in varying degrees. But conscious creation and direction, the consciousness of acts, is limited to the highestlevel psycho-neural activity-the self.

I have suggested above that, when the physiological activities of the lower-level systems meet with the highest-level activities, they may become manifest as conscious presentations; these highestlevel activities are, I believe, to be regarded as arising from the supreme organisation and distillation of the directive activities of the living organism. The acts at this highest mental level constitute the purposeful, directive, creative, and contemplative self, and are the recipients of presentations from lower cortical, and also of feelings from lower, primordial, thalamic activity.

The psychologist's principle of the conservation of self, which corresponds to the biologist's inevitable principle of the struggle for existence, is the fundamental function of this conscious activity. It is as real and important as the physicist's principle of conservation of energy. We must leave to the future the task of bridging the present impassable gulf which yawns between these two principles. Meanwhile let us always remember that blind mechanism in the material world is a truth not more fundamental than the reign of guidance, creation, and purpose in the world of life and mind, and, it may well be, throughout the universe; indeed, our very notions of these two principles, governing perhaps both the living and lifeless world, appear to be the outcome of, even if they do not wholly depend upon, the experiencing, reasoning, and imagining self. 